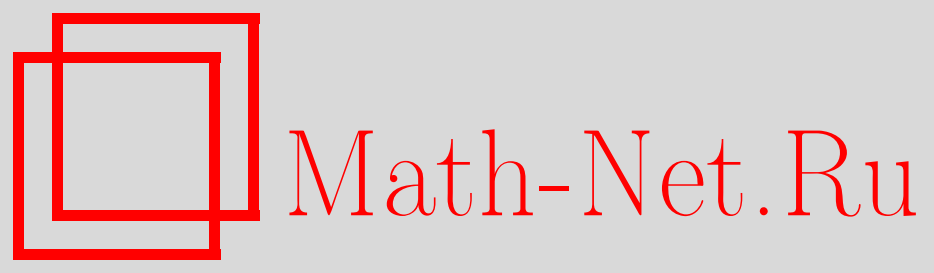

В. В. Кочергин, О некоторых мерах сложности конечных абелевых групп, Дискрет. матем., 2015, том 27, выпуск 3, 25-43

DOI: https://doi.org/10.4213/dm1333

Использование Общероссийского математического портала Math-Net.Ru подразумевает, что вы прочитали и согласны с пользовательским соглашением http://www.mathnet.ru/rus/agreement

Параметры загрузки:

IP : 52.90 .164 .192

26 апреля 2023 г., 14:32:17

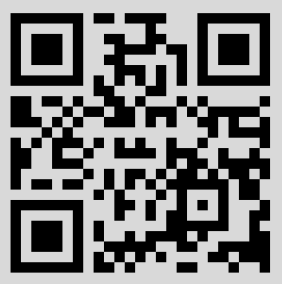




\title{
О некоторых мерах сложности конечных абелевых групп
}

\author{
() 2015 г. В. В. Кочергин*
}

Пусть конечная абелева мультипликативная группа $G$ задана базисом $B=\left\{a_{1}, a_{2}, \ldots, a_{q}\right\}$, т. е. группа $G$ раскладывается в прямое произведение циклических подгрупп, порожденных элементами множества $B: G=$ $\left\langle a_{1}\right\rangle \times\left\langle a_{2}\right\rangle \times \ldots \times\left\langle a_{q}\right\rangle$. Сложность $L(g ; B)$ элемента $g$ группы $G$ в базисе $B$ определяется как минимальное число операций умножения, достаточное для вычисления элемента $g$, исходя из элементов базиса $B$ (разрешается многократное использование результатов промежуточных вычислений). Пусть $L(G, B)=\max _{g \in G} L(g ; B), L M(G)=\max _{B} L(G, B), L m(G)=\min _{B} L(G, B), M(n)=$ $\max _{G:|G| \leqslant n} L M(G), m(n)=\max _{G:|G| \leqslant n} L m(G), M_{\mathrm{Cp}}(n)=\left(\sum_{G:|G|=n} L M(G)\right) / A(n)$, $m_{\mathrm{cp}}(n)=\left(\sum_{G:|G|=n} L m(G)\right) / A(n)$, где $A(n)-$ количество абелевых групп порядка $n$. В работе получены асимптотические оценки величин $L(G, B), M(n)$, $m(n), M_{\mathrm{cp}}(n)$ и $m_{\mathrm{cp}}(n)$.

Работа выполнена при финансовой поддержке РФФИ, проект № 14-01-00598.

Ключевые слова: конечная абелева группа, сложность вычисления, аддитивные цепочки, векторные аддитивные цепочки, задача Беллмана, задача Кнута.

\section{1. Постановка задачи}

Пусть $G$ - конечная абелева группа (групповую операцию будем называть умножением). Подмножество $B=\left\{a_{1}, \ldots, a_{q}\right\}$ элементов группы будем называть базисом в группе $G$, если $G$ раскладывается в прямое произведение циклических подгрупп, порожденных элементами множества $B$ :

$$
G=\left\langle a_{1}\right\rangle_{u_{1}} \times \ldots \times\left\langle a_{q}\right\rangle_{u_{q}}
$$

где $u_{i}$ - порядок элемента $a_{i}, i=1, \ldots, q$.

Для каждого элемента $g$ группы $G$ определим его сложность реализации над базисом $B$, обозначаемую через $L(g ; B)$ как минимальное число операций умножения,

* Место работы: МГУ им. М. В. Ломоносова, ИТПМ им. Н.Н. Боголюбова МГУ, e-mail: vvkoch@yandex.ru 
достаточное для вычисления элемента $g$ с использованием элементов множества $B$, при этом все уже вычисленные элементы могут быть использованы многократно в этом принципиальное отличие этой, «схемной», меры сложности от другой, «формульной» (см., например, [2]), меры сложности вычислений элементов в группах. Отметим также, что в алгебре под задачей вычислений в группе понимают, как правило, совсем другую задачу, а именно, задачу распознавания равенства слов в группе - см., например, [14].

Сложность реализации элемента $g$ группы $G$ над базисом $B$ удобно интерпретировать на языке схем из функциональных элементов [13], как это сделано, например, в [5]: на входы схем подаются базисные элементы группы $G$, сами схемы состоят из двухвходовых элементов, которые по двум представителям группы $G$, поступающим на входы, реализуют их произведение; под сложностью схемы понимается число функциональных элементов в схеме (т. е. операций умножения), а сложность реализации $L(g ; B)$ элемента $g$ группы $G$ над базисом $B$ численно равна минимальной сложности схем, реализующих элемент $g$ над базисом $B$.

В данной работе имеет смысл дать еще одно эквивалентное определение величины $L(g ; B)$.

Въчислительной цепочкой (по аналогии с аддитивной цепочкой [3]) для элемента $g$ конечной абелевой группы $G$ над базисом $B=\left\{a_{1}, \ldots, a_{q}\right\}$ будем называть всякую последовательность $a_{1}, \ldots, a_{q}, h_{1}, h_{2}, \ldots, h_{r}=g$ элементов группы $G$, удовлетворяющую условию: для каждого $k, 1 \leqslant k \leqslant r$, найдутся два такие элемента (не обязательно различные) $h_{k 1}$ и $h_{k 2}$ из множества $\left\{a_{1}, \ldots, a_{q}, h_{1}, h_{2}, \ldots, h_{k-1}\right\}$ (т. е. лежащие в этой последовательности левее элемента $\left.g_{k}\right)$, что $h_{k}=h_{k 1} h_{k 2}$. Число $r$ называется длиной вычисления элемента $g$ над базисом $B$, минимальная длина вычисления элемента $g$ над базисом $B$ совпадает с величиной $L(g ; B)$ и называется сложностью реализации (вычисления) элемента $g$ над базисом $B$.

Сложность $L(G, B)$ конечной абелевой группь $G$ над базисом $B$ определим так:

$$
L(G, B)=\max _{g \in G} L(g ; B) .
$$

Положим

$$
L M(G)=\max _{B: \text { - базис } G} L(G, B), \quad L m(G)=\min _{B: \text { - базис } G} L(G, B) .
$$

Так как конечная абелева группа $G$ полностью определяется вектором $\mathbf{v}=$ $\left(v_{1}, \ldots, v_{q}\right)$ порядков примарных циклических подгрупп группы $G$, то вместо обозначения $L M(G)$ можно использовать обозначение $M(\mathbf{v})$, а вместо $L m(G)-m(\mathbf{v})$.

Для абелевой группы, порядки примарных циклических подгрупп которой задаются вектором $\mathbf{v}$, будем использовать обозначение $G_{\mathbf{v}}$.

Для вектора $\mathbf{v}=\left(v_{1}, \ldots, v_{q}\right)$ обозначим через $\|\mathbf{v}\|$ величину $v_{1} v_{2} \ldots v_{q}$. Положим

$$
M(n)=\max _{\mathbf{v}:\|\mathbf{v}\| \leqslant n} M(\mathbf{v}), \quad m(n)=\max _{\mathbf{v}:\|\mathbf{v}\| \leqslant n} m(\mathbf{v}) .
$$

Кроме того, введем функции $M_{\mathrm{cp}}(n)$ и $m_{\mathrm{cp}}(n)$, характеризующие средние значения соответствующих мер сложности абелевых групп порядка $n$, определив их равенствами

$$
M_{\mathrm{cp}}(n)=\frac{\sum L M(G)}{A(n)}, \quad m_{\mathrm{cp}}(n)=\frac{\sum L m(G)}{A(n)},
$$


где суммы берутся по всем различным (с точностью до изоморфизма) абелевым группам $G$ порядка $n$, а $A(n)$ - количество попарно неизоморфных абелевых групп порядка $n$.

Задача заключается в том, чтобы, во-первых, найти числовые функции $f_{1}(\mathbf{v})$ и $f_{2}(\mathbf{v})$, определенные на векторах $\mathbf{v}$, характеризующих порядки примарных циклических групп, с помощью которых выражались бы величины $M(\mathbf{v})$ и $m(\mathbf{v})$ (хотя бы асимптотически или с точностью до порядка при условии, что порядок всей группы стремится к бесконечности); во-вторых, исследовать рост функций $M(n)$ и $m(n)$, а также функций $M_{\mathrm{cp}}(n)$ и $m_{\mathrm{cp}}(n)$, при $n \rightarrow \infty$.

Именно в таком виде, по-видимому, весной 1988 года Олег Борисович Лупанов ставил автору, тогда еще студенту-пятикурснику, задачу о сложности вычисления элементов конечных абелевых групп. С тех пор в этом и близких направлениях был получен ряд результатов, постановки задач видоизменялись, расширялись, переосмысливались, так или иначе все дальше удаляясь от исходной. Однако данная работа является попыткой по возможности наиболее полно ответить как раз на изначально поставленные вопросы. Часть изложенных здесь результатов сформулирована в $[10,11]$.

\section{2. Известные результаты и близкие задачи}

Нахождение точных формул для введенных мер сложности вычислений в конечных абелевых группах представляется нереальной задачей (что подтверждается, в частности, $N P$-полнотой близкой задачи [22], о которой ниже также пойдет речь). Поэтому далее будем говорить только об асимптотических (с ростом порядка группы) оценках (по возможности асимптотически точных или хотя бы точных по порядку).

Достаточно серьезное продвижение в решении этих задач было получено в работе [5] (см. также краткий вариант [4]). Помимо двух простых нижних оценок (одна из которых стандартная мощностная) сложности конечной абелевой группы над заданным базисом была получена и важная верхняя оценка. Сформулируем эти результаты в виде утверждений. Для этого введем следующие обозначения.

Пусть $G=\left\langle a_{1}\right\rangle_{u_{1}} \times \ldots \times\left\langle a_{q}\right\rangle_{u_{q}}$. Тогда для базиса $B=\left\{a_{1}, \ldots, a_{q}\right\}$ положим $k(B)=\max _{1 \leqslant i \leqslant q} u_{i}$. Кроме того, для произвольного базиса $B$ конечной абелевой группы положим $q(B)=|B|$. Иногда будем вместо $q(B)$ использовать обозначение $q$ (если при этом не возникает коллизий).

Утверждение 1 [5]. Пусть $B-$ базис конечной абелевой группы $G$. Тогда ${ }^{1}$

$$
L(G, B) \geqslant\lfloor\log (k(B)-1)\rfloor+q(B)-1 .
$$

Утверждение 2 [5]. Для произвольного положительного в найдется такое положительное $m(\varepsilon)$, что для сложности любой конечной абелевой группь $G$ над базисом В при выполнении условия $|G|>m(\varepsilon)$ справедлива оченка

$$
L(G, B) \geqslant \frac{\log |G|}{\log \log |G|}\left(1+(1-\varepsilon) \frac{\log \log \log |G|}{\log \log |G|}\right) .
$$

\footnotetext{
13десь и далее все логарифмы берутся по основанию 2.
} 
Утверждение 3 [5]. Существуют такие положительные константы $c_{1} u c_{2}$, что для произвольной конечной абелевой группы $G$ и любого базиса $B$ этой группь справедливо неравенство

$$
L(G, B) \leqslant \frac{\log |G|}{\log \log |G|}\left(1+c_{1}\left(\frac{\log \log \log |G|}{\log \log |G|}\right)^{1 / 2}\right)+c_{2} \max (\log k(B), q(B)) .
$$

Здесь стоит отметить, что извлекаемые из доказательства данного утверждения значения констант $c_{1}$ и $c_{2}$ довольно велики.

Тем не менее утверждения 1-3 устанавливают порядок роста величины $L(G, B)$ : при условии $|G| \rightarrow \infty$ справедливо равенство

$$
L(G, B)=\Theta\left(\frac{\log |G|}{\log \log |G|}+\log k(B)+q(B)\right) .
$$

Кроме того, в работе [5] на основе уже выписанных утверждений исследован аимптотический рост функции $L(n)$, определяемой равенством $L(n)=\max L M(G)$, где максимум берется по всем абелевым группам $G$ порядка $n$.

Утверждение 4 [5]. При $n \rightarrow \infty$ справедливо равенство

$$
L(n)=\log n+\frac{\log n}{\log \log n}+o\left(\frac{\log n}{\log \log n}\right) .
$$

Тем самым фактически была решена задача об асимптотике роста величины $M(n)$ (несколько подробнее об этом будет сказано ниже).

Собственно, на этом результаты в данном направлении формально, по-видимому, и исчерпываются. Были еще две работы автора $[6,9]$, являющиеся в некотором смысле логическим продолжением статьи [5], но они посвящены задачам сложности вычислений (в том же самом смысле) в конечных нильпотентных и разрешимых группах, и в них новых результатов для класса конечных абелеых групп практически не содержится. Однако задачи, поставленные О. Б. Лупановым, имеют тесную связь с задачей Ричарда Беллмана, на которой остановимся несколько подробнее.

В 1963 г. Р. Беллман [21] (для случая $m=2$ ), а затем в 1964 г. Е. Страус [26] (для произвольного $m$ ) сформулировали задачу о нахождении сложности вычисления одночлена от $m$ переменных. Под сложностью вычисления одночлена $x_{1}^{n_{1}} x_{2}^{n_{2}} \ldots x_{m}^{n_{m}}$ (обозначение $\left.l\left(x_{1}^{n_{1}} x_{2}^{n_{2}} \ldots x_{m}^{n_{m}}\right)\right)$ понимается минимальное число операций умножения, достаточное для вычисления по переменным $x_{1}, x_{2}, \ldots, x_{m}$ одночлена $x_{1}^{n_{1}} x_{2}^{n_{2}} \ldots x_{m}^{n_{m}}$. На аддитивном языке величина $l\left(x_{1}^{n_{1}} x_{2}^{n_{2}} \ldots x_{m}^{n_{m}}\right)$ формально определяется как минимально возможная длина $r$ последовательности $m$-мерных векторов (наборов)

$$
\begin{aligned}
\mathbf{d}_{1}=(1,0, \ldots, 0), \mathbf{d}_{2}=(0,1, \ldots, 0), \ldots, \mathbf{d}_{q}=(0,0, \ldots, 1), \\
\\
\mathbf{d}_{q+1}, \mathbf{d}_{q+2}, \ldots, \mathbf{d}_{q+r}=\left(n_{1}, n_{2}, \ldots, n_{m}\right),
\end{aligned}
$$

начинающейся с $m$ единичных векторов и удовлетворяющей условию: для каждого $k(q+1 \leqslant k \leqslant q+r)$ найдутся два таких натуральных числа (не обязательно различных) $i$ и $j, 1 \leqslant i \leqslant k-1,1 \leqslant j \leqslant k-1$, что $\mathbf{d}_{k}=\mathbf{d}_{i}+\mathbf{d}_{j}$ (сложение векторов покомпонентное). 
На самом деле задача Беллмана эквивалентна $[16,23,24]$ известной задаче Дональда Кнута [3; разд. 4.6.3, упр. 32] о нахождении величины $l\left(x^{n_{1}}, x^{n_{2}}, \ldots, x^{n_{m}}\right)-$ сложности вычисления набора степеней $\left(x^{n_{1}}, x^{n_{2}}, \ldots, x^{n_{m}}\right)$, т. е. минимального числа умножений, достаточного для вычисления множества степеней $x^{n_{1}}, x^{n_{2}}, \ldots, x^{n_{m}}$ исходной переменной $x$. Такая эквивалентность, заключающаяся в справедливости равенства

$$
l\left(x_{1}^{n_{1}} x_{2}^{n_{2}} \ldots x_{m}^{n_{m}}\right)+1=l\left(x^{n_{1}}, x^{n_{2}}, \ldots, x^{n_{m}}\right)+m,
$$

позволяет говорить об этих двух задачах как об одной задаче Беллмана-Кнута.

Небольшой обзор результатов по задаче Беллмана - Кнута можно найти в [12].

Очевидно, что для произвольного элемента $a_{1}^{n_{1}} a_{2}^{n_{2}} \ldots a_{m}^{n_{m}}$ абелевой группы $\left\langle a_{1}\right\rangle \times$ $\left\langle a_{2}\right\rangle \times \ldots \times\left\langle a_{m}\right\rangle$ справедливо неравенство

$$
L\left(a_{1}^{n_{1}} a_{2}^{n_{2}} \ldots a_{m}^{n_{m}},\left\{a_{1}, a_{2}, \ldots, a_{m}\right\}\right) \leqslant l\left(x_{1}^{n_{1}} x_{2}^{n_{2}} \ldots x_{m}^{n_{m}}\right) .
$$

Поэтому верхняя оценка сложности в задаче Беллмана - Кнута автоматически дает верхнюю оценку в задаче о сложности вычисления элементов конечных абелевых групп. С получением нижних оценок сложности вычисления элементов конечных абелевых групп через нижние оценки для задачи Беллмана - Кнута, конечно, все не так однозначно. Приведем два примера.

Пример 1. С одной стороны, справедливо равенство $l\left(x^{31}\right)=7$ (см., например, [3]), а с другой стороны, в группе $\langle a\rangle_{33}$, очевидно, выполняется соотношение $L\left(a^{31},\{a\}\right)=6$.

Обобщая, получаем, с одной стороны, неравенство

$$
l\left(x^{2^{n}-1}\right) \geqslant n+\log n-2,13,
$$

вытекающее из основного результата статьи [25], а с другой, для группы $\langle a\rangle_{2^{n}+1}-$ равенство

$$
L\left(a^{2^{n}-1},\{a\}\right)=n+1
$$

Таким образом,

$$
l\left(x^{2^{n}-1}\right)-L\left(a^{2^{n}-1},\{a\}\right) \geqslant \log n-3,13 .
$$

Пример 2. Для произвольного $m$ обозначим через $\left(n_{1}, n_{2}, \ldots, n_{m}\right)$ набор, удовлетворяющий двум условиям:

1) $n_{i} \leqslant 2^{m}, i=1,2, \ldots, m$;

2) $l\left(x_{1}^{n_{1}} x_{2}^{n_{2}} \ldots x_{m}^{n_{m}}\right)=\max l\left(x_{1}^{t_{1}} x_{2}^{t_{2}} \ldots x_{m}^{t_{m}}\right)$, где максимум берется по всем наборам $\left(t_{1}, t_{2}, \ldots, t_{m}\right)$ с целыми неотрицательными компонентами, не превышающими величины $2^{m}$.

Из стандартных мощностных соображений (см., например, [7]) при всех достаточно больших значениях $m$ следует такое неравенство:

$$
l\left(x_{1}^{n_{1}} x_{2}^{n_{2}} \ldots x_{m}^{n_{m}}\right) \geqslant \frac{m^{2}}{2 \log m} .
$$


Теперь положим $n=\max _{j=1,2, \ldots, m} n_{j}, r_{i}=2 n+1-n_{i}(i=1,2, \ldots, m)$. Отметим, что при всех $i, 1 \leqslant i \leqslant m$, выполняются неравенства $n_{i}<r_{i}$. Рассмотрим группу $\left\langle a_{1}\right\rangle_{r_{1}} \times\left\langle a_{2}\right\rangle_{r_{2}} \times \ldots \times\left\langle a_{m}\right\rangle_{r_{m}}$. В этой группе справедлива цепочка равенств

$$
\left(a_{1} a_{2} \ldots a_{m}\right)^{2 n+1}=\prod_{i=1}^{m} a_{i}^{2 n+1-n_{i}+n_{i}}=\prod_{i=1}^{m}\left(a_{i}^{r_{i}} a_{i}^{n_{i}}\right)=a_{1}^{n_{1}} a_{2}^{n_{2}} \ldots a_{m}^{n_{m}} .
$$

Поэтому, используя известный факт о том, что для возведения в степень $t$ достаточно $\log t+o(\log t)$ операций умножения [20] (см. также, например, [3]), при $m \rightarrow \infty$ получаем следующую оценку² :

$$
L\left(a_{1}^{n_{1}} a_{2}^{n_{2}} \ldots a_{m}^{n_{m}},\left\{a_{1}, a_{2}, \ldots a_{m}\right\}\right) \leqslant \log n+o(\log n)+m \lesssim 2 m .
$$

Следовательно,

$$
l\left(x_{1}^{n_{1}} x_{2}^{n_{2}} \ldots x_{m}^{n_{m}}\right) \gtrsim \frac{\left(L\left(a_{1}^{n_{1}} a_{2}^{n_{2}} \ldots a_{m}^{n_{m}},\left\{a_{1}, a_{2}, \ldots a_{m}\right\}\right)\right)^{2}}{8 \log L\left(a_{1}^{n_{1}} a_{2}^{n_{2}} \ldots a_{m}^{n_{m}},\left\{a_{1}, a_{2}, \ldots a_{m}\right\}\right)},
$$

т. е. отличие в порядках роста сложности с увеличением параметра $m$ для двух задач почти квадратичное.

Таким образом, сами нижние оценки, полученные при исследовании задачи Беллмана - Кнута, не могут быть напрямую применены для получения нижних оценок сложности вычислений элементов конечных абелевых групп. Однако многие методы получения нижних оценок с различной степенью эффективности работают и в этой задаче.

Возвращаясь к верхним оценкам в задаче Беллмана-Кнута, отметим, что наиболее сильная из них получена в работе [12] на основе оценок из [1], которые, в свою очередь, существенно опираются на результаты из [5]. Нам достаточно будет следующей упрощенной формулировки (в работе [12] это следствие к теореме 1).

Утверждение 5 [12]. Для любой последовательности наборов натуральньх чисел $\tilde{n}(s)=\left(n_{1}(s), n_{2}(s), \ldots, n_{m(s)}(s)\right), s=1,2, \ldots$, удовлетворяющей условию

$$
\sum_{i=1}^{m(s)} n_{i}(s) \rightarrow \infty
$$

при $s \rightarrow \infty$ выполняется неравенство

$$
l\left(x_{1}^{n_{1}} x_{2}^{n_{2}} \ldots x_{m}^{n_{m}}\right) \lesssim \log \left(\max _{1 \leqslant i \leqslant m} n_{i}(s)\right)+\frac{\log N(s)}{\log \log N(s)}+m(s),
$$

где $N(s)=n_{1}(s) n_{2}(s) \ldots n_{m}(s)$.

\footnotetext{
2 Для функций $f(m)$ и $g(m)$, заданных на множестве натуральных чисел, при $m \rightarrow \infty$ запись $f(m) \lesssim g(m)$ означает выполнение условия $\varlimsup_{m \rightarrow \infty} \frac{f(m)}{g(m)} \leqslant 1$, запись $f(m) \gtrsim g(m)-$ выполнение условия $\underline{\lim }_{m \rightarrow \infty} \frac{f(m)}{g(m)} \geqslant 1$, и, наконец, запись $f(m) \sim g(m)-$ выполнение условия $\lim _{m \rightarrow \infty} \frac{f(m)}{g(m)}=1$.
} 
С использованием утверждения 5 один из результатов работы [5] можно значительно усилить следующим образом.

Теорема 1. Пусть $G=\left\langle a_{1}\right\rangle_{u_{1}} \times \ldots \times\left\langle a_{q}\right\rangle_{u_{q}}, B=\left\{a_{1}, \ldots, a_{q}\right\}$. Тогда при $|G| \rightarrow \infty$ справедливы соотношения

$$
\max \left(\frac{\log |G|}{\log \log |G|}, \log \left(\max _{i} u_{i}\right)+q\right) \lesssim L(G, B) \lesssim \frac{\log |G|}{\log \log |G|}+\log \left(\max _{i} u_{i}\right)+q .
$$

Теперь, возвращаясь к нижним оценкам, сформулируем в упрощенном виде, удобном для наших целей, основной результат работы [8].

Утверждение 6 [8]. Пусть последовательность наборов

$$
\tilde{n}(s)=\left(n_{1}(s), n_{2}(s), \ldots, n_{m(s)}(s)\right), \quad s=1,2, \ldots,
$$

натуральных чисел при $s \rightarrow \infty$ удовлетворяет условию

$$
m(s)=o\left(\log \left(\max _{i} n_{i}(s)\right)+\frac{\log N(s)}{\log \log N(s)}\right),
$$

где $N(s)=\prod_{i=1}^{m(s)} n_{i}(s)$. Тогда при $s \rightarrow \infty$ справедливо соотношение

$$
\max l\left(x_{1}^{k_{1}} x_{2}^{k_{2}} \ldots x_{m(s)}^{k_{m(s)}}\right) \gtrsim \log \left(\max _{i} n_{i}(s)\right)+\frac{\log N(s)}{\log \log N(s)},
$$

где максимум берется по всем наборам $\left(k_{1}, k_{2}, \ldots, k_{m(s)}\right)$ иельх чисел, удовлетворяющих условиям $0 \leqslant k_{i} \leqslant n_{i}(s), i=1,2, \ldots, m(s)$.

Как уже отмечалось выше, из нижней оценки для задачи Беллмана напрямую не следует аналогичная нижняя оценка сложности конечных абелевых групп. Тем не менее, почти дословно повторяя рассуждения из работы [8], можно установить следующий факт.

Теорема 2. Пусть $B-$ базис конечной абелевой группы $G$. Если $|G| \rightarrow \infty$ и въполняется условие

$$
q(B)=o\left(\frac{\log |G|}{\log \log |G|}+\log k(B)\right)
$$

то справедлива асимптотическая оценка

$$
L(G, B) \gtrsim \frac{\log |G|}{\log \log |G|}+\log k(B) .
$$

\section{3. Основные результаты}

Изложение результатов стоит начать с формулировки ответа на первую часть задачи - по большому счету он фактически содержится в [5]. 
Теорема 3. При $n \rightarrow \infty$ справедливы равенства

$$
\begin{aligned}
& M(n)=\log n+\frac{\log n}{\log \log n}+o\left(\frac{\log n}{\log \log n}\right) ; \\
& m(n)=\log n+\frac{\log n}{\log \log n}+o\left(\frac{\log n}{\log \log n}\right) .
\end{aligned}
$$

Доказательство. Справедливость первого равенства следует из утверждения 4 и соотношения

$$
M(n)=\max _{1 \leqslant m \leqslant n} L(m) .
$$

Верхняя оценка второго равенства следует из первого равенства и неравенства $m(n) \leqslant M(n)$. Докажем нижнюю оценку. Воспользуемся теоремой Бертрана - Чебышёва о распределении простых чисел (см., например, [18]), в силу которой найдется простое $p$, удовлетворяющее условиям $\lceil n / 2\rceil<p<2\lceil n / 2\rceil$, а следовательно, и неравенствам $n / 2<p \leqslant n$. Тогда

$$
m(n) \geqslant L\left(\langle a\rangle_{p},\{a\}\right)
$$

а доказательство соотношения

$$
L\left(\langle a\rangle_{m},\{a\}\right)-\log m \gtrsim \frac{\log m}{\log \log m}
$$

при $m \rightarrow \infty$ содержится в доказательстве нижней оценки теоремы 5 из [5], по существу и составляя это доказательство.

Теорема 3 доказана.

Тем самым решена одна из поставленных О.Б. Лупановым задач - найдена асимптотика роста функций $M(n)$ и $m(n)$ (на самом деле не только асимптотика, но и оценка остаточного члена).

Теперь перейдем к исследованию асимптотики роста величин $M(\mathbf{v})$ и $m(\mathbf{v})$.

Функцию $h(n)$ натурального аргумента будем называть допустимой, если выполняется следующее свойство - для любых двух последовательностей натуральных чисел $\left\{d_{n}^{(1)}\right\}$ и $\left\{d_{n}^{(2)}\right\}$, удовлетворяющих условиям:

1) $d_{n}^{(1)} \rightarrow \infty$

2) $d_{n}^{(2)} / 2 \leqslant d_{n}^{(1)} \leqslant 2 d_{n}^{(2)}$ для всех достаточно больших значений $n$, при $n \rightarrow \infty$ справедливо асимптотическое соотношение $h\left(d_{n}^{(1)}\right) \sim h\left(d_{n}^{(2)}\right)$.

Заметим, что замена коэффициентов $1 / 2$ и 2 , фигурирующих во втором условии определения, на произвольные константы $1 / c$ и $c$, где $c>1$, приводит к эквивалентному определению допустимой функции. Поэтому допустимую функцию можно определить как функцию натурального аргумента, допускающую доопределение по медленно меняющейся на бесконечности функции (см., например, [15]).

Теорема 4. При $\|\mathbf{v}\| \rightarrow \infty$ выполняются соотношения

$$
\frac{\log \|\mathbf{v}\|}{\log \log \|\mathbf{v}\|} \lesssim m(\mathbf{v}) \leqslant M(\mathbf{v}) \lesssim \log \|\mathbf{v}\|,
$$


причем для любых допустимых функиий $h_{1}(n)$ u $h_{2}(n)$, удовлетворяющих при $n \rightarrow$ $\infty$ условиям

$$
\frac{\log n}{\log \log n} \lesssim h_{1}(n) \lesssim h_{2}(n) \lesssim \log n,
$$

найдется последовательность векторов $\mathbf{v}_{s}$, удовлетворяющая условиям $\left\|\mathbf{v}_{s}\right\| \rightarrow \infty$

$$
m\left(\mathbf{v}_{s}\right) \sim h_{1}\left(\left\|\mathbf{v}_{s}\right\|\right), \quad M\left(\mathbf{v}_{s}\right) \sim h_{2}\left(\left\|\mathbf{v}_{s}\right\|\right) .
$$

Доказательство. Асимптотическое неравенство

$$
m(\mathbf{v}) \gtrsim \frac{\log \|\mathbf{v}\|}{\log \log \|\mathbf{v}\|}
$$

следует непосредственно из утверждения 2 , соотношения $m(\mathbf{v}) \leqslant M(\mathbf{v}) \leqslant M(\|\mathbf{v}\|)$ очевидны, а для завершения доказательства цепочки соотношений из утверждения теоремы остается применить теорему 3.

Теперь установим существование требуемой последовательности.

Без ограничения общности можно считать, что функции $h_{1}(n)$ и $h_{2}(n)$ при всех достаточно больших $n$ удовлетворяют неравенствам

$$
\frac{\log n}{\log \log n} \leqslant h_{1}(n) \leqslant h_{2}(n) \leqslant \log n+\frac{\log n}{\log \log n} .
$$

Действительно, если это не так, то от функций $h_{1}(n)$ и $h_{2}(n)$ можно перейти к функциям $\hat{h}_{1}(n)$ и $\hat{h}_{2}(n)$, определяемым следующим образом:

$$
\begin{aligned}
& \hat{h}_{1}(n)=\max \left\{\frac{\log n}{\log \log n}, \min \left\{h_{1}(n), h_{2}(n), \log n+\frac{\log n}{\log \log n}\right\}\right\} \\
& \hat{h}_{2}(n)=\min \left\{\max \left\{\frac{\log n}{\log \log n}, h_{1}(n), h_{2}(n)\right\}, \log n+\frac{\log n}{\log \log n}\right\} .
\end{aligned}
$$

Функции $\hat{h}_{1}(n)$ и $\hat{h}_{2}(n)$ асимптотически равны функциям $h_{1}(n)$ и $h_{2}(n)$ соответственно, являются допустимыми и удовлетворяют соотношениям

$$
\frac{\log n}{\log \log n} \leqslant \hat{h}_{1}(n) \leqslant \hat{h}_{2}(n) \leqslant \log n+\frac{\log n}{\log \log n} .
$$

Положим

$$
\begin{gathered}
t=t(s)=\left\lceil\max \left\{h_{1}\left(2^{s}\right)-\frac{s}{\log s}, \frac{s}{(\log s)^{2}}\right\}\right\rceil, \\
d_{1}=d_{1}(s)=\left\lceil\frac{s-\left\lfloor h_{2}\left(2^{s}\right)-h_{1}\left(2^{s}\right)\right\rfloor}{t}\right\rceil .
\end{gathered}
$$

Тогда, с одной стороны, справедливо неравенство $d_{1} \leqslant(\log s)^{2}+1$, а с другой, в силу соотношений

$$
s-\left\lfloor h_{2}\left(2^{s}\right)-h_{1}\left(2^{s}\right)\right\rfloor \geqslant s-h_{2}\left(2^{s}\right)+h_{1}\left(2^{s}\right) \geqslant h_{1}\left(2^{s}\right)-\frac{s}{\log s},
$$

выполняется условие $d_{1} \geqslant 1$.

2 Дискретная математика, т. 27 № 3 
Пусть $p_{1}$ - максимальное простое число, не превосходящее $2^{\left\lceil s /(\log s)^{2}\right\rceil}, p_{2}-$ предшествующее числу $p_{1}$ простое число (т. е. $p_{2}$ - максимальное простое число, меньшее $\left.p_{1}\right), p_{3}$ - предшествующее числу $p_{2}$ простое число и т. д. Определим параметр $d_{2}=d_{2}(s)$ из условия

$$
p_{1} p_{2} \ldots p_{d_{2}-1}<2^{\left\lfloor h_{2}\left(2^{s}\right)-h_{1}\left(2^{s}\right)\right\rfloor} \leqslant p_{1} p_{2} \ldots p_{d_{2}-1} p_{d_{2}} .
$$

Установим корректность определения $d_{2}$. В силу теоремы Бертрана - Чебышёва о распределении простых чисел (см., например, [18]) выполняются неравенства

$$
p_{i} \geqslant \frac{2^{\left\lceil s /(\log s)^{2}\right\rceil}}{2^{i}}, \quad i=1,2, \ldots\left\lceil s /(\log s)^{2}\right\rceil-1 .
$$

Поэтому при $j=\left\lceil 2(\log s)^{2}\right\rceil$ справедливы соотношения

$$
\prod_{i=1}^{j} p_{i} \geqslant \frac{2^{j\left\lceil s /(\log s)^{2}\right\rceil}}{2^{1} 2^{2} \ldots 2^{j}} \geqslant 2^{j s /(\log s)^{2}-j(j+1) / 2}>2^{s} \geqslant 2^{\left\lfloor h_{2}\left(2^{s}\right)-h_{1}\left(2^{s}\right)\right\rfloor} .
$$

Следовательно, значение $d_{2}$ с требуемыми свойствами существует, причем $d_{2} \leqslant$ $\left\lceil 2(\log s)^{2}\right\rceil$.

Теперь определим вектор $\mathbf{v}_{s}=\left(v_{1}(s), \ldots, v_{d_{1}+d_{2}}(s)\right)$, положив

$$
\begin{gathered}
v_{1}=v_{2}=\ldots=v_{d_{1}-1}=2^{t}, \quad v_{d_{1}}=2^{s-\left\lfloor h_{2}\left(2^{s}\right)-h_{1}\left(2^{s}\right)\right\rfloor-\left(d_{1}-1\right) t}, \\
v_{d_{1}+1}=p_{1}, v_{d_{1}+2}=p_{2}, \ldots, v_{d_{1}+d_{2}-1}=p_{d_{2}-1}, \quad v_{d_{1}+d_{2}}=p_{0},
\end{gathered}
$$

где $p_{0}$ - наименьшее из простых чисел $p$, отличных от 2 и удовлетворяющих условию

$$
p_{1} p_{2} \ldots p_{d_{2}-1} p \geqslant 2^{\left\lfloor h_{2}\left(2^{s}\right)-h_{1}\left(2^{s}\right)\right\rfloor} .
$$

Тогда выполняются соотношения

$$
2^{s} \leqslant\left\|\mathbf{v}_{s}\right\| \leqslant 3 \cdot 2^{s}, \quad d_{1}+d_{2} \leqslant 4(\log s)^{2} \leqslant 4\left(\log \log \left\|\mathbf{v}_{s}\right\|\right)^{2} .
$$

Поэтому для любого базиса $B$ группы $G_{\mathbf{v}_{s}}$ выполняется неравенство $q(B) \leqslant$ $\left(\log \log \left\|\mathbf{v}_{s}\right\|\right)^{2}$ и в силу теорем 1 и 2 справедливо асимптотическое соотношение

$$
L\left(G_{\mathbf{v}_{s}}, B\right) \sim \frac{\log \left\|\mathbf{v}_{s}\right\|}{\log \log \left\|\mathbf{v}_{s}\right\|}+\log k(B)
$$

при $s \rightarrow \infty$.

Таким образом при $s \rightarrow \infty$ имеем:

$$
\begin{gathered}
m\left(\mathbf{v}_{s}\right) \sim \frac{\log \left\|\mathbf{v}_{s}\right\|}{\log \log \left\|\mathbf{v}_{s}\right\|}+\log v_{1} \sim \\
\sim \frac{s}{\log s}+\max \left\{h_{1}\left(2^{s}\right)-\frac{s}{\log s}, \frac{s}{(\log s)^{2}}\right\} \sim h_{1}\left(2^{s}\right) \sim h_{1}\left(\left\|\mathbf{v}_{s}\right\|\right), \\
M\left(\mathbf{v}_{s}\right) \sim \frac{\log \left\|\mathbf{v}_{s}\right\|}{\log \log \left\|\mathbf{v}_{s}\right\|}+\log \left(v_{1} v_{d_{1}+1} v_{d_{1}+2} \ldots v_{d_{1}+d_{2}}\right) \sim \\
\sim \frac{s}{\log s}+\max \left\{h_{1}\left(2^{s}\right)-\frac{s}{\log s}, \frac{s}{(\log s)^{2}}\right\}+h_{2}\left(2^{s}\right)-h_{1}\left(2^{s}\right) \sim h_{2}\left(2^{s}\right) \sim h_{2}\left(\left\|\mathbf{v}_{s}\right\|\right) .
\end{gathered}
$$

Теорема 4 доказана. 
Перейдем к более подробному исследованию величины $M(\mathbf{v})$. Пусть группа $G$ представлена как прямое произведение своих примарных циклических компонент:

$$
G=\left\langle a_{1}\right\rangle_{v_{1}} \times \ldots \times\left\langle a_{q}\right\rangle_{v_{q}} .
$$

Обозначим через $q(\mathbf{v})$ размерность (число координат) вектора $\mathbf{v}$. Далее для каждого простого делителя $p_{i}$ величины $\|\mathbf{v}\|$ обозначим через $P_{i}(\mathbf{v})$ максимальный из порядков примарных циклических подгрупп, являющихся степенями числа $p_{i}$. Теперь положим $P(\mathbf{v})=\prod P_{i}(\mathbf{v})$, где произведение берется по всем простым делителям числа $\|\mathbf{v}\|$. Легко заметить, что величина $P(\mathbf{v})$ численно равна максимальному значению порядка среди всех элементов группы $G$. Кроме того, всегда найдется базис $B_{1}$ группы $G$, для которого справедливо равенство $k\left(B_{1}\right)=P(\mathbf{v})$.

Очевидно, что справедливы оценки $M(\mathbf{v}) \geqslant q(\mathbf{v})-1$ и $M(\mathbf{v}) \geqslant \log (P(\mathbf{v})-1)$, но «объединить» их в одно неравенство подобно утверждению 1 не удается. Однако можно доказать следующую оценку.

Теорема 5. При $\|\mathbf{v}\| \rightarrow \infty$ справедливо асимптотическое неравенство

$$
M(\mathbf{v}) \gtrsim q(\mathbf{v})+\log P(\mathbf{v}) .
$$

Доказательство. Рассмотрим отдельно несколько случаев.

Случай 1. Пусть выполняется неравенство

$$
q(\mathbf{v}) \leqslant \frac{\log P(\mathbf{v})}{\log \log \log \|\mathbf{v}\|} .
$$

Тогда

$$
M(\mathbf{v}) \geqslant \log (P(\mathbf{v})-1) \gtrsim q(\mathbf{v})+\log P(\mathbf{v}) .
$$

Случай 2. Пусть выполняется неравенство

$$
\log P(\mathbf{v}) \leqslant \frac{q(\mathbf{v})}{\log \log \log \|\mathbf{v}\|} .
$$

Тогда

$$
M(\mathbf{v}) \geqslant q(\mathbf{v})-1 \gtrsim q(\mathbf{v})+\log P(\mathbf{v}) .
$$

Случай 3. Пусть выполняются неравенства

$$
q(\mathbf{v}) \geqslant \frac{\log P(\mathbf{v})}{\log \log \log \|\mathbf{v}\|}, \quad \log P(\mathbf{v}) \geqslant \frac{q(\mathbf{v})}{\log \log \log \|\mathbf{v}\|} .
$$

Рассмотрим базис $B^{\prime}$, имеющий среди всех базисов $B$, удовлетворяющих равенству $k(B)=P(\mathbf{v})$, наибольшее значение величины $q(B)$.

Случай 3.1. Пусть выполняется неравенство

$$
q(\mathbf{v})-q\left(B^{\prime}\right) \leqslant \frac{q(\mathbf{v})}{\log \log \log \|\mathbf{v}\|}
$$

Тогда, обозначив абелеву группу, задаваемую вектором $\mathbf{v}$, через $G$, получаем:

$$
M(\mathbf{v}) \geqslant L\left(G, B^{\prime}\right) \geqslant q\left(B^{\prime}\right)+\log \left(k\left(B^{\prime}\right)-1\right)-1 \geqslant
$$




$$
\geqslant q(\mathbf{v})+\log (P(\mathbf{v})-1)-\frac{q(\mathbf{v})}{\log \log \log \|\mathbf{v}\|}-1 \sim q(\mathbf{v})+\log P(\mathbf{v}) .
$$

Случай 3.2. Пусть выполняется неравенство

$$
q(\mathbf{v})-q\left(B^{\prime}\right)>\frac{q(\mathbf{v})}{\log \log \log \|\mathbf{v}\|}
$$

Тогда в силу определения базиса $B^{\prime}$, вектор, составленный из порядков элементов базиса $B^{\prime}$, выглядит (с точностью до перестановки координат) следующим образом. Величина одной из координат равна $k\left(B^{\prime}\right)=P(\mathbf{v})$, для оставшихся $q\left(B^{\prime}\right)-1$ координат существует взаимно однозначное отображение на $q\left(B^{\prime}\right)-1$ координат вектора $\mathbf{v}$, сохраняющее величины координат, при этом величины оставшихся $q(\mathbf{v})-q\left(B^{\prime}\right)+1$ координат вектора $\mathbf{v}$ попарно различны. Поэтому

$$
P(\mathbf{v})=k\left(B^{\prime}\right) \geqslant \prod_{i=1}^{q(\mathbf{v})-q\left(B^{\prime}\right)+1}(i+1)
$$

Следовательно,

$$
\begin{aligned}
\log P(\mathbf{v})=k\left(B^{\prime}\right) & \geqslant \sum_{i=1}^{q(\mathbf{v})-q\left(B^{\prime}\right)+1} \log (i+1) \geqslant \frac{q(\mathbf{v})-q\left(B^{\prime}\right)}{2} \log \frac{q(\mathbf{v})-q\left(B^{\prime}\right)}{2} \geqslant \\
& \geqslant \frac{1}{4} \frac{q(\mathbf{v})}{\log \log \log \|\mathbf{v}\|}(\log q(\mathbf{v})-\log \log \log \log \|\mathbf{v}\|) .
\end{aligned}
$$

Тогда справедливо неравенство

$$
q(\mathbf{v}) \leqslant 2^{5(\log \log \log \|\mathbf{v}\|)^{2}} .
$$

Действительно, если это не так, то из предыдущей цепочки соотношений следует, что при всех достаточно больших значениях $\|\mathbf{v}\|$ выполняется неравенство

$$
\log P(\mathbf{v})>q(\mathbf{v}) \log \log \log \|\mathbf{v}\|,
$$

что противоречит условиям случая 3 .

Таким образом, применяя теорему 4 и учитывая условия случая 3, при $\|\mathbf{v}\| \rightarrow \infty$ получаем:

$$
M(\mathbf{v}) \gtrsim \frac{\log \|\mathbf{v}\|}{\log \log \|\mathbf{v}\|}=\frac{2^{2^{\log \log \log \|\mathbf{v}\|}}}{2^{\log \log \log \|\mathbf{v}\|}}>q(\mathbf{v})+q(\mathbf{v}) \log \log \log \|\mathbf{v}\| \geqslant q(\mathbf{v})+\log P(\mathbf{v}) .
$$

Теорема 5 доказана.

Следующая теорема дает ответ еще на одну часть исходной задачи.

Теорема 6. При $\|\mathbf{v}\| \rightarrow \infty$ выполняются соотношения

$$
\max \left(\frac{\log \|\mathbf{v}\|}{\log \log \|\mathbf{v}\|}, q(\mathbf{v})+\log P(\mathbf{v})\right) \lesssim M(\mathbf{v}) \lesssim \frac{\log \|\mathbf{v}\|}{\log \log \|\mathbf{v}\|}+q(\mathbf{v})+\log P(\mathbf{v}) .
$$


Доказательство. Нижняя оценка непосредственно следует из утверждения 2 и предыдущей теоремы (теоремы 5). Для доказательства верхней оценки обозначим для группы $G$, определяемой вектором $\mathbf{v}$, через $B_{M}$ базис, удовлетворяющий условию $M(\mathbf{v})=L\left(G, B_{M}\right)$. Тогда с использованием верхней оценки теоремы 1 и неравенств $k\left(B_{M}\right) \leqslant P(\mathbf{v})$ и $q\left(B_{M}\right) \leqslant q(\mathbf{v})$ имеем:

$M(\mathbf{v})=L\left(G, B_{M}\right) \lesssim \frac{\log |G|}{\log \log |G|}+\log k\left(B_{M}\right)+q\left(B_{M}\right) \leqslant \frac{\log \|\mathbf{v}\|}{\log \log \|\mathbf{v}\|}+q(\mathbf{v})+\log P(\mathbf{v})$.

Теорема 6 доказана.

Эта теорема уставливает порядок роста величины $M(\mathbf{v})$, причем верхняя оценка может превышать нижнюю асимптотически не более чем в два раза. Кроме того, в случае, когда одна из величин $\frac{\log \|\mathbf{v}\|}{\log \log \|\mathbf{v}\|}$ или $q(\mathbf{v})+\log P(\mathbf{v})$ растет существенно быстрее другой, т. е. выполняется условие

$$
\frac{\log \|\mathbf{v}\|}{\log \log \|\mathbf{v}\|(\log P(\mathbf{v})+q(\mathbf{v}))}+\frac{\log \log \|\mathbf{v}\|(\log P(\mathbf{v})+q(\mathbf{v}))}{\log \|\mathbf{v}\|} \rightarrow \infty,
$$

теорема 6 устанавливает следующую асимптотику роста функционала сложности $M(\mathbf{v})$ :

$$
M(\mathbf{v}) \sim \frac{\log \|\mathbf{v}\|}{\log \log \|\mathbf{v}\|}+\log P(\mathbf{v})+q(\mathbf{v})
$$

Для формулировки оценок асимптотического роста величины $m(\mathbf{v})$ введем обозначения. Пусть $B$ - базис конечной абелевой группы $G$. Положим

$$
r(B)=\lfloor\log (k(B)-1)\rfloor+q(B), \quad r(\mathbf{v})=\underset{B: B-\text { базис } G_{\mathbf{v}}}{\min } r(B) .
$$

Теорема 7. С одной стороны, при $\|\mathbf{v}\| \rightarrow \infty$ имеет место верхняя оценка

$$
m(\mathbf{v}) \lesssim \frac{\log \|\mathbf{v}\|}{\log \log \|\mathbf{v}\|}+r(\mathbf{v})
$$

с другой стороны, при всех достаточно больших значениях $\|\mathbf{v}\|$ справедлива нижняя оценка

$$
m(\mathbf{v}) \geqslant \max \left(\frac{\log \|\mathbf{v}\|}{\log \log \|\mathbf{v}\|}, r(\mathbf{v})-1\right) .
$$

Доказательство. Нижняя оценка непосредственно следует из утверждений 1 и 2 , а верхняя - из верхней оценки теоремы 1.

Теорема 7 доказана.

Эта теорема уставливает порядок роста величины $m(\mathbf{v})$, причем опять верхняя оценка может превышать нижнюю асимптотически не более чем в два раза. В случае, когда одна из величин $\frac{\log \|\mathbf{v}\|}{\log \log \|\mathbf{v}\|}$ или $r(\mathbf{v})$ растет существенно быстрее другой, т. е. выполняется условие

$$
\frac{\log \|\mathbf{v}\|}{(\log \log \|\mathbf{v}\|) r(\mathbf{v})}+\frac{(\log \log \|\mathbf{v}\|) r(\mathbf{v})}{\log \|\mathbf{v}\|} \rightarrow \infty,
$$


теорема 7 устанавливает следующую асимптотику роста функционала сложности $m(\mathbf{v})$ :

$$
m(\mathbf{v}) \sim \frac{\log \|\mathbf{v}\|}{\log \log \|\mathbf{v}\|}+r(\mathbf{v})
$$

В заключение исследуем асимптотическое поведение функций $M_{\mathrm{cp}}(n)$ и $m_{\mathrm{cp}}(n)$, характеризующих средние значения соответствующих мер сложности абелевых групп порядка $n$.

Теорема 8. При $n \rightarrow \infty$ выполняются соотношения

$$
\frac{\log n}{\log \log n} \lesssim m_{c p}(n) \leqslant M_{c p}(n) \lesssim \log n,
$$

причем для любых допустимых функций $h_{1}(n)$ u $h_{2}(n)$, удовлетворяющих при $n \rightarrow$ $\infty$ условиям

$$
\frac{\log n}{\log \log n} \lesssim h_{1}(n) \lesssim h_{2}(n) \lesssim \log n
$$

найдется последовательность $\left\{n_{s}\right\}$, удовлетворяющая условию $n_{s} \rightarrow \infty$ nри $s \rightarrow$ $\infty$, для которой справедливы соотношения

$$
m_{c p}\left(n_{s}\right) \sim h_{1}\left(n_{s}\right), \quad M_{c p}\left(n_{s}\right) \sim h_{2}\left(n_{s}\right) .
$$

Доказательство. Универсальные соотношения из первой части теоремы непосредственно следуют из теоремы 4, поэтому сразу перейдем к доказательству существования требуемой последовательности.

Как и при доказательстве теоремы 4, без ограничения общности можно считать, что функции $h_{1}(n)$ и $h_{2}(n)$ при всех достаточно больших $n$ удовлетворяют неравенствам

$$
\frac{\log n}{\log \log n} \leqslant h_{1}(n) \leqslant h_{2}(n) \leqslant \log n+\frac{\log n}{\log \log n} .
$$

Положим

$$
\begin{aligned}
& H_{1}=H_{1}(s)=\left\lceil\max \left\{h_{1}\left(2^{s}\right)-\frac{s}{\log s}, \frac{s}{(\log s)^{2}}\right\}\right], \\
& H_{2}=H_{2}(s)=\left\lceil\max \left\{h_{2}\left(2^{s}\right)-\frac{s}{\log s}, \frac{s}{(\log s)^{2}}\right\}\right\rceil .
\end{aligned}
$$

Отметим, что выполняется неравенство $H_{2} \leqslant s$.

Пусть $p_{1}$ - максимальное простое число, меньшее $2^{H_{1}}, p_{2}$ - предшествующее числу $p_{1}$ простое число (т. е. $p_{2}$ - максимальное простое число, меньшее $\left.p_{1}\right), p_{3}-$ предшествующее числу $p_{2}$ простое число и т. д. Определим параметр $d=d(s)$ условием

$$
p_{1} p_{2} \ldots p_{d-1}<2^{H_{2}} \leqslant p_{1} p_{2} \ldots p_{d-1} p_{d} .
$$

Установим корректность определения $d$. В силу теоремы Бертрана - Чебышёва о распределении простых чисел (см., например, [18]) выполняются неравенства

$$
p_{i} \geqslant \frac{2^{\left\lceil s /(\log s)^{2}\right\rceil}}{2^{i}}, \quad i=1,2, \ldots\left\lceil s /(\log s)^{2}\right\rceil-1 .
$$


Поэтому при $j=\left\lceil 2(\log s)^{2}\right\rceil$ справедливы соотношения

$$
\prod_{i=1}^{j} p_{i} \geqslant \frac{2^{j\left\lceil s /(\log s)^{2}\right\rceil}}{2^{1} 2^{2} \ldots 2^{j}} \geqslant 2^{j s /(\log s)^{2}-j(j+1) / 2}>2^{s} \geqslant 2^{H_{2}} .
$$

Следовательно, значение $d$ с требуемыми свойствами существует, причем $d \leqslant$ $\left\lceil 2(\log s)^{2}\right\rceil$.

Отметим также справедливость неравенства $d \geqslant 2$, вытекающего из соотношений $p_{1}<2^{H_{1}} \leqslant 2^{H_{2}}$.

Обозначим через $p_{d}^{\prime}$ наименьшее из простых чисел $x$, отличных от 2 и удовлетворяющих условию

$$
p_{1} p_{2} \ldots p_{d-1} x \geqslant 2^{H_{2}} .
$$

Поэтому справедливы неравенства

$$
2^{H_{2}} \leqslant p_{1} p_{2} \ldots p_{d-1} p_{d}^{\prime}<3 \cdot 2^{H_{2}} .
$$

Теперь положим

$$
\begin{gathered}
t=t(s)=s-H_{2}, \\
n_{s}=2^{t} p_{1} p_{2} \ldots p_{d-1} p_{d}^{\prime} .
\end{gathered}
$$

Тогда выполняются неравенства

$$
2^{s} \leqslant n_{s}<3 \cdot 2^{s}
$$

В силу теоремы 2 имеем:

$$
\begin{gathered}
m_{\mathrm{cp}}\left(n_{s}\right) \gtrsim \frac{\log n_{s}}{\log \log n_{s}}+\log p_{1}, \\
M_{\mathrm{cp}}\left(n_{s}\right) \gtrsim \frac{\log n_{s}}{\log \log n_{s}}+\log \left(p_{1} p_{2} \ldots p_{d-1} p_{d}^{\prime}\right) .
\end{gathered}
$$

Перейдем к получению верхних оценок величин $m_{\mathrm{cp}}\left(n_{s}\right)$ и $M_{\mathrm{cp}}\left(n_{s}\right)$.

Отметим, что число всех попарно неизоморфных абелевых групп порядка $n_{s}$ равно числу всех попарно неизоморфных абелевых групп порядка $2^{t(s)}$ и, в свою очередь, равно числу неупорядоченных разбиений числа $t(s)$ на натуральные слагаемые (см., например, $[17,19])$. Обозначим это число через $\varrho(t(s))$. Для произвольной абелевой группы $G$ порядка $n_{s}$ обозначим через $B_{G}^{m}$ некоторый базис этой группы, удовлетворяющий условию $L m(G)=L\left(G, B_{G}^{m}\right)$, а через $B_{G}^{M}-$ некоторый базис, удовлетворяющий условию $L M(G)=L\left(G, B_{G}^{M}\right)$. Тогда

$$
m_{\mathrm{cp}}\left(n_{s}\right)=\frac{\sum L\left(G, B_{G}^{m}\right)}{\varrho(t(s))}, \quad M_{\mathrm{cp}}\left(n_{s}\right)=\frac{\sum L\left(G, B_{G}^{M}\right)}{\varrho(t(s))},
$$

где суммы берутся по всем $\varrho(t(s))$ различным (с точностью до изоморфизма) абелевым группам $G$ порядка $n_{s}$.

Для оценки величин $L\left(G, B_{G}^{m}\right)$ и $L\left(G, B_{G}^{M}\right)$ используем справедливую при $|G| \rightarrow \infty$ верхнюю оценку из теоремы 1 :

$$
L(G, B) \lesssim \frac{\log |G|}{\log \log |G|}+\log k(B)+q(B) .
$$


Рассмотрим два случая.

Случай 1. Пусть выполняется неравенство $t \leqslant \frac{\log n_{s}}{\left(\log \log n_{s}\right)^{2}}$. Тогда для любой группы $G$ порядка $n_{s}$ при $s \rightarrow \infty$ выполняются соотношения

$$
\begin{gathered}
L\left(G, B_{G}^{m}\right) \lesssim \frac{\log n_{s}}{\log \log n_{s}}+\log \left(2^{t} p_{1}\right)+(t+d) \sim \frac{\log n_{s}}{\log \log n_{s}}+\log p_{1}, \\
L\left(G, B_{G}^{M}\right) \lesssim \frac{\log n_{s}}{\log \log n_{s}}+\log \left(2^{t} p_{1} p_{2} \ldots p_{d-1} p_{d}^{\prime}\right)+(t+d) \sim \\
\sim \frac{\log n_{s}}{\log \log n_{s}}+\log \left(p_{1} p_{2} \ldots p_{d-1} p_{d}^{\prime}\right) .
\end{gathered}
$$

Поэтому

$$
m_{\mathrm{cp}}\left(n_{s}\right) \lesssim \frac{\log n_{s}}{\log \log n_{s}}+\log p_{1}, \quad M_{\mathrm{cp}}\left(n_{s}\right) \lesssim \frac{\log n_{s}}{\log \log n_{s}}+\log \left(p_{1} p_{2} \ldots p_{d-1} p_{d}^{\prime}\right) .
$$

Случай 2. Пусть выполняется неравенство $t>\frac{\log n_{s}}{\left(\log \log n_{s}\right)^{2}}$. Тогда $t \rightarrow \infty$ при $s \rightarrow \infty$.

Обозначим через $a(t)$ число различных (с точностью до изоморфизма) абелевых групп порядка $2^{t}$, у которых есть примарная компонента порядка не менее $2^{t /(\log t)^{2}}$, а через $b(t)$ - число различных (с точностью до изоморфизма) абелевых групп порядка $2^{t}$, у которых число примарных компонент не менее $t /(\log t)^{2}$.

Тогда, учитывая, что $t \leqslant s \leqslant \log n_{s}$ и $d \leqslant\left\lceil 2(\log s)^{2}\right\rceil$, можно выписать такие оценки:

$$
\begin{gathered}
M_{\mathrm{cp}}\left(n_{s}\right) \lesssim \frac{\log n_{s}}{\log \log n_{s}}+\log \left(2^{t /(\log t)^{2}} p_{1} p_{2} \ldots p_{d-1} p_{d}^{\prime}\right)+\left(\frac{t}{(\log t)^{2}}+d\right)+ \\
+\frac{\log \left(2^{t}\right) a(t)+t b(t)}{\varrho(t)} \sim \frac{\log n_{s}}{\log \log n_{s}}+\log \left(p_{1} p_{2} \ldots p_{d-1} p_{d}^{\prime}\right)+\frac{\operatorname{ta}(t)+t b(t)}{\varrho(t)}, \\
m_{\mathrm{cp}}\left(n_{s}\right) \lesssim \frac{\log n_{s}}{\log \log n_{s}}+\log \left(2^{t /(\log t)^{2}} p_{1}\right)+\left(\frac{t}{(\log t)^{2}}+d\right)+\frac{\log \left(2^{t}\right) a(t)+t b(t)}{\varrho(t)} \sim \\
\sim \frac{\log n_{s}}{\log \log n_{s}}+\log p_{1}+\frac{\operatorname{ta}(t)+t b(t)}{\varrho(t)} .
\end{gathered}
$$

Отметим, что величина $a(t)$ равна числу неупорядоченных разбиений числа $t$ на натуральные слагаемые, наибольшее из которых не менее $t /(\log t)^{2}$, а величина $b(t)$ равна числу неупорядоченных разбиений числа $t$ на не менее $t /(\log t)^{2}$ натуральных слагаемых. Известно (см., например, теорему 4.1.1 из [17]), что число разбиений натурального числа $t$ на $u$ частей равно числу разбиений $t$ на части, наибольшая из которых есть $u$. Поэтому $a(t)=b(t)$.

Далее, для $i=1,2, \ldots, t$ обозначив через $\varrho_{i}(t)$ число неупорядоченных разбиений числа $t$ на $i$ частей, имеем:

$$
b(t)=\sum_{i=\left\lceil t /(\log t)^{2}\right\rceil}^{t} \varrho_{i}(t) \leqslant \sum_{i=\left\lceil t /(\log t)^{2}\right\rceil}^{t} \varrho(t-i)<t \varrho\left(t-\left\lceil t /(\log t)^{2}\right\rceil\right) .
$$


Теперь, используя асимптотическую формулу (при $n \rightarrow \infty)$

$$
\varrho(n) \sim \frac{1}{4 \sqrt{3} n} e^{\pi \sqrt{\frac{2 n}{3}}}
$$

которая следует из более сильной теоремы Харди-Рамануджана (см., например, $[17,19])$, получаем:

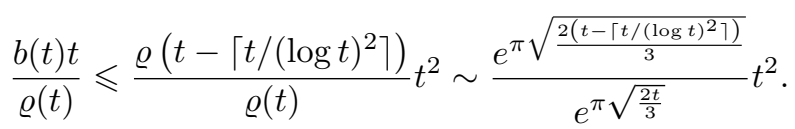

Покажем, что последнее выражение стремится к 0 при $t \rightarrow \infty$. Действительно, логарифм этой величины стремится к $-\infty$ при $t \rightarrow \infty$ :

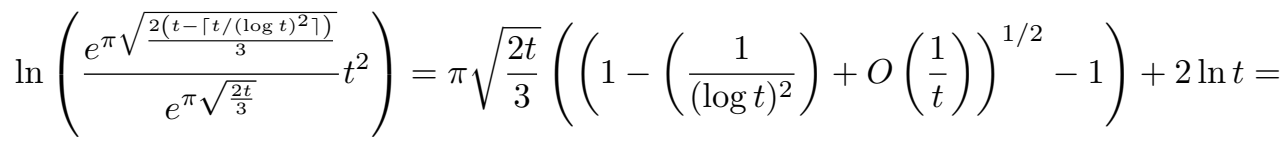

$$
\begin{aligned}
& =\pi \sqrt{\frac{2 t}{3}}\left(-\left(\frac{1}{2(\log t)^{2}}\right)+O\left(\frac{1}{(\log t)^{4}}\right)\right)+2 \ln t=-\pi \sqrt{\frac{2}{12}} \frac{\sqrt{t}}{(\log t)^{2}}+O\left(\frac{\sqrt{t}}{(\log t)^{4}}\right) .
\end{aligned}
$$

Таким образом,

$$
\frac{t a(t)+t b(t)}{\varrho(t)}=o(1)
$$

и, следовательно, в случае 2 справедливы также верхние оценки

$$
m_{\mathrm{cp}}\left(n_{s}\right) \lesssim \frac{\log n_{s}}{\log \log n_{s}}+\log p_{1}, \quad M_{\mathrm{cp}}\left(n_{s}\right) \lesssim \frac{\log n_{s}}{\log \log n_{s}}+\log \left(p_{1} p_{2} \ldots p_{d-1} p_{d}^{\prime}\right)
$$

Объединяя верхние оценки с нижними оценками, доказанными ранее, и учитывая допустимость функций $h_{1}$ и $h_{2}$, получаем:

$$
\begin{aligned}
m_{\mathrm{cp}}\left(n_{s}\right) \sim \frac{\log n_{s}}{\log \log n_{s}} & +\log p_{1} \sim \\
& \sim \frac{s}{\log s}+\max \left\{h_{1}\left(2^{s}\right)-\frac{s}{\log s}, \frac{s}{(\log s)^{2}}\right\} \sim h_{1}\left(2^{s}\right) \sim h_{1}\left(n_{s}\right), \\
M_{\mathrm{cp}}\left(n_{s}\right) \sim \frac{\log n_{s}}{\log \log n_{s}} & +\log \left(p_{1} p_{2} \ldots p_{d-1} p_{d}^{\prime}\right) \sim \\
& \sim \frac{s}{\log s}+\max \left\{h_{2}\left(2^{s}\right)-\frac{s}{\log s}, \frac{s}{(\log s)^{2}}\right\} \sim h_{2}\left(2^{s}\right) \sim h_{2}\left(n_{s}\right) .
\end{aligned}
$$

Теорема 8 доказана. 


\section{Список литературы}

1. Гашков С. Б., Кочергин В. В., “Об аддитивных цепочках векторов, вентильных схемах и сложности вычисления степеней", Методы дискретного анализа в теории графов и сложности, 52, Новосибирск, 1992, 22-40.

2. Глухов М. М., Зубов А. Ю., "О длинах симметрических и знакопеременных групп подстановок в различных системах образующих", Математические вопросы кибернетики, 8, Наука, Москва, 1999, 5-32.

3. Кнут Д. Е., Искусство программирования для ЭВМ, 2, Мир, Москва, 1977.

4. Кочергин В. В., "О сложности вычислений в конечных абелевых группах", Доклады AH CCCP, 317:2 (1991), 291-294.

5. Кочергин В. В., "О сложности вычислений в конечных абелевых группах", Математические вопросы кибернетики, 4, Наука, Москва, 1992, 178-217.

6. Кочергин В. В., "О сложности вычислений в конечных абелевых, нильпотентных и разрешимых группах", Дискретная математика, 5:1 (1993), 91-111.

7. Кочергин В. В., "О вычислении наборов степеней", Дискретная математика, 6:2 (1994), 129-137.

8. Кочергин В. В., "О сложности вычислений одночленов и наборов степеней", Дискретный анализ (Тр./РАН. Сиб. отделение. Ин-т математики; Т. 27), Издательство Института математики СО РАН, Новосибирск, 1994, 94-107.

9. Кочергин В. В., "О сложности вычислений в конечных нильпотентных группах", Дискретный анализ и исследование операций, 3:1 (1996), 43-51.

10. Кочергин В. В., "Некоторые задачи сложности вычисления элементов конечных абелевых групп", Материаль ХІ Междунар. сем. "Дискретная математика и ее приложения» (Москва, МГУ, 18-23 июня 2012 г.), Изд-во мех.-мат. ф-та МГУ, Москва, 2012, $135-138$.

11. Кочергин В. В., "Об одной нижней оценке сложности вычисления элементов конечных абелевых групп”, Проблемы теоретической кибернетики. Материалы XVII междунар. конф. (Казань, 16-20 июня 2014 г.), Отечество, Казань, 2014, 155-158.

12. Кочергин В. В., "Уточнение оценок сложности вычисления одночленов и наборов степеней в задачах Беллмана и Кнута", Дискретный анализ и исследование операций, 21:6 (2014), 51-72.

13. Лупанов О. Б., "О синтезе некоторых классов управляющих систем”, Проблемы кибернетики, 10, Физматгиз, Москва, 1963, 63-97.

14. Ольшанский А. Ю., "О сложности вычислений в группах", Соросовский образовательный журнал, 6:3 (2000), 118-123.

15. Сенета Е., Правильно меняющиеся функиии, М.: Наука, 1985, 144 с.

16. Сидоренко А. Ф., "Сложность аддитивных вычислений семейств целочисленных линейных форм", Зап. научн. сем. ЛОМИ, 105, Наука, Ленинград, 1981, 53-61.

17. Холл М., Комбинаторика, Мир, Москва, 1970.

18. Чандрасекхаран К., Введение в аналитическую теорию чисел, Мир, Москва, 1974.

19. Эндрюс Г., Теория разбиений, Наука, Москва, 1982.

20. Brauer A., "On addition chains", Bull. Amer. Math. Soc., 45 (1939), 736-739.

21. Bellman R. E., "Addition chains of vectors (Advanced problem 5125)", Amer. Math. Monthly, 70 (1963), 765.

22. Downey P., Leong B., Sethi R., "Computing sequences with addition chains", SIAM J. Comput., 10 (1981), 638-646.

23. Knuth D. E., Papadimitriou C. H., "Duality in addition chains", Bull. Ass. Theor. Comput. Sci., 13 (1981), 2-4.

24. Olivos J., "On vectorial addition chains", J. Algorithms, 2:1 (1981), 13-21. 
25. Schönhage A., "A lower bound for the lenght of addition chains", Theor. Comput. Sci., 1 (1975), 1-12.

26. Straus E. G., "Addition chains of vectors", Amer. Math. Monthly, 71 (1964), 806-808.

Статья поступила 25.05.2015. 